\section{Решение транспортной задачи в среде Excel-7.0}

\author{
Ю. Н. Кондратьев ${ }^{1}$ \\ Петрозаводский государственный университет
}

\section{АННОТАЦИЯ}

Статья посвящена оптимизации транспортных перевозок в среде Excel-7. Приведен конкретный пример решения транспортной задачи.

Ключевые слова: транспортная задача, среда Excel-7.

\section{SUMMARY}

The article is devoted to the problem of transport optimization in software Excel-7. It presents a specific example of the transport problem solution.

Keywords: transport task, optimization, software Excel-7.

Решение транспортной задачи, которая связана с оптимизацией перевозок различных грузов, является актуальной задачей.

В настоящее время эта задача решается при помощи программирования на различных алгоритмических языках. В то же время решение этой задачи можно осуществлять более простыми методами в среде Excel-7.0.

Известно, что транспортная задача в общем виде имеет следующую формулировку:

1. Имеется т пунктов отправления (ПО) $\mathrm{A}_{1}, \mathrm{~A}_{2}, \ldots, \mathrm{A}_{\mathrm{m}}$, в которых сосредоточены запасы каких-то однородных грузов в количестве соответственно $\mathrm{a}_{1}, \mathrm{a}_{2}, \ldots, \mathrm{a}_{\mathrm{m}}$ единиц.

2. Имеется $\mathrm{n}$ пунктов назначения (ПН) $\mathrm{B}_{1}, \mathrm{~B}_{2}, \ldots, \mathrm{B}_{\mathrm{n}}$, подавших заявки на получение соответственно $\mathrm{b}_{1}$, $\mathrm{b}_{2}, \ldots, \mathrm{b}_{\mathrm{n}}$ единиц груза.

3. Сумма всех запасов грузов равна сумме всех заявок (равенство 1):

$$
\sum_{\mathrm{i}=1}^{\mathrm{m}} \mathrm{a}_{\mathrm{i}}=\sum_{\mathrm{j}=1}^{\mathrm{n}} \mathrm{b}_{\mathrm{j}} \text {, }
$$

где $\mathrm{i}=1,2, \ldots, \mathrm{m}$, a $\mathrm{j}=1,2, \ldots, \mathrm{n}$.

4. Известны стоимости $\mathrm{C}_{\mathrm{i}, \mathrm{j}}$ перевозки единицы груза от каждого пункта отправления $\mathrm{A}_{\mathrm{i}}$ до каждого пункта назначения $\mathrm{B}_{\mathrm{j}}$.

Bсе числа стоимости $\mathrm{C}_{\mathrm{i}, \mathrm{j}}$ перевозки единицы груза образуют прямоугольную матрицу (2):

\footnotetext{
${ }^{1}$ Автор - доцент кафедры технологии металлов и ремонта

(C) Ю. Н. Кондратьев, 2003
}

$$
\begin{aligned}
& \mathrm{C}_{1,1} \mathrm{C}_{1,2} \ldots \mathrm{C}_{1, \mathrm{n}} \\
& \mathrm{C}_{2,1} \mathrm{C}_{2,2} \ldots \mathrm{C}_{2, \mathrm{n}} \\
& \ldots \ldots \ldots \ldots \ldots \ldots \\
& \mathrm{C}_{\mathrm{m}, 1} \mathrm{C}_{\mathrm{m}, 2} \ldots \mathrm{C}_{\mathrm{m}, \mathrm{n}}
\end{aligned}
$$

5. Требуется составить такой план перевозок (откуда, куда и сколько единиц груза), чтобы все заявки были выполнены, а общая стоимость всех перевозок была бы минимальной.

Для решения этой задачи обозначим количество перевозимого груза $\mathrm{X}_{\mathrm{i}, \mathrm{j}}$

Тогда неотрицательные значения этих переменных можно записать в виде матрицы (3).

$X_{1,1} X_{1,2} \ldots X_{1, n}$
$X_{2,1} X_{2,2} \ldots X_{2, n}$
$\ldots \ldots \ldots \ldots \ldots \ldots$
$X_{m, 1} X_{m, 2} \ldots X_{m, n}$

В свою очередь неотрицательные переменные должны удовлетворять следующим условиям:

1) Суммарное количество груза, вывозимого из каждого ПО во все ПН, должно быть равно запасу груза в данном пункте, тогда это даст m уравнений-равенств (4):

$\mathrm{X}_{1,1}+\mathrm{X}_{1,2}+\ldots+\mathrm{X}_{1, \mathrm{n}}=\mathrm{a}_{1}$

$\mathrm{X}_{2,1}+\mathrm{X}_{2,2}+\ldots+\mathrm{X}_{2, \mathrm{n}}=\mathrm{a}_{2}$

$\mathrm{X}_{\mathrm{m}, 1}+\mathrm{X}_{\mathrm{m}, 2}+\ldots+\mathrm{X}_{\mathrm{m}, \mathrm{n}}=\mathrm{a}_{\mathrm{m}}$

2) Суммарное количество груза, поступающее в каждый ПН из всех ПО, должно соответствовать заявке каждого пункта назначения, тогда это даст $\mathbf{n}$ условий-равенств (5):

$\mathrm{X}_{1,1}+\mathrm{X}_{2,1}+\ldots+\mathrm{Xm}, 1_{1}=\mathrm{b}_{1}$

$\mathrm{X}_{1,2}+\mathrm{X}_{2,2}+\ldots+\mathrm{X}_{\mathrm{m}, 2}=\mathrm{b}_{2}$

$\mathrm{X}_{1, \mathrm{n}}+\mathrm{X}_{2, \mathrm{n}}+\ldots+\mathrm{X}_{\mathrm{m}, \mathrm{n}}=\mathrm{b}_{\mathrm{n}}$

Тогда целевая функция примет вид (6):

$Z=\sum_{i=1}^{m} \cdot \sum_{j=1}^{n} C_{i, j} \cdot X_{i, j} \Rightarrow \min$

Особенностью транспортной задачи является то, что все коэффициенты при неизвестных $\mathrm{X}_{\mathrm{i}, \mathrm{j}}$ в условиях (4) и (5) равны единицам.

В данной задаче число линейно независимых уравнений равно числу базисных переменных:

$\mathrm{lu}=\mathrm{Bp}=\mathrm{m}+\mathrm{n}-1$

где lu - число линейно независимых уравнений; Вр - число базисных переменных, а число свободных переменных равно: 
$\mathrm{Sp}=\mathrm{m} * \mathrm{n}-(\mathrm{m}+\mathrm{n}-1)=(\mathrm{m}-1) *(\mathrm{n}-1)$.

(8)

Следовательно, в оптимальном плане число $(\mathrm{m}-1)(\mathrm{n}-1)$ перевозок будет равно нулю, то есть из каких-то пунктов отправления в какие-то пункты назначения ничего не будет перевозиться.

Решение транспортной задачи в среде Excel-7.0 рассмотрим на конкретном примере с исходными данными, приведенными в табл. 1, то есть имеется три пункта отправления $\mathrm{A}_{\mathrm{i}}$ с количеством груза в каждом пункте 35,45 и 50 единиц.

Требуется перевезти эти грузы в четыре пункта назначения $\mathrm{B}_{\mathrm{j}}$ с заявками $30,10,65$ и 25 единиц со стоимостью перевозок единицы груза из $\mathrm{A}_{\mathrm{i}}$ в $\mathrm{B}_{\mathrm{j}}$, представленной в табл. 1.

Для решения задачи в электронную таблицу вводятся исходные данные и записываются условия (табл. 2).

Таблица 1

Условия задачи

\begin{tabular}{|c|c|c|c|c|c|}
\hline \multicolumn{2}{|c|}{ Пункт отправления (ПО) } & \multicolumn{4}{|c|}{ Пункт назначения (ПН) } \\
\hline \multirow{2}{*}{ обозначение } & \multirow{2}{*}{ запас груза } & $\mathrm{B}_{1}$ & $\mathrm{~B}_{2}$ & $\mathrm{~B}_{3}$ & $\mathrm{~B}_{4}$ \\
\cline { 2 - 6 } & & & \multicolumn{4}{|c|}{ Стоимость перевозок $\mathrm{C}_{\mathrm{i}, \mathrm{j}}$, у.е. } & $\mathbf{6}$ & $\mathbf{1 1}$ \\
\hline $\mathrm{A}_{1}$ & 35 & $\mathbf{5}$ & $\mathbf{1 3}$ & $\mathbf{1 2}$ & $\mathbf{8}$ \\
\hline $\mathrm{A}_{2}$ & 45 & $\mathbf{4}$ & $\mathbf{7}$ & $\mathbf{3}$ & $\mathbf{1 0}$ \\
\hline $\mathrm{A}_{3}$ & 50 & $\mathbf{9}$ & $\mathbf{2}$ & 65 & 25 \\
\hline \multicolumn{2}{|c|}{ Заявка } & 30 & 10 &
\end{tabular}

Таблица 2

\begin{tabular}{|c|c|c|}
\hline \multicolumn{2}{|c|}{ Запись условий в ячейку } & \multirow{2}{*}{$\begin{array}{c}\text { Запись условий в окно } \\
\text { Поиск решения }\end{array}$} \\
\hline адрес ячейки & условия & \\
\hline G13 & СУММПРОИЗВ(В7:Е7;В19:Е19) & $\$ \mathrm{G} \$ 13=\$ \mathrm{I} \$ 13$ \\
\hline G14 & СУММПРОИЗВ(В8:Е8;В20:Е20) & $\$ G \$ 14=\$ I \$ 14$ \\
\hline G15 & СУММПРОИЗВ(В9:Е9;В21:Е21) & $\$ \mathrm{G} \$ 15=\$ \mathrm{I} \$ 15$ \\
\hline G16 & СУММ(B7:B9) & $\$ \mathrm{G} \$ 16=\$ \mathrm{I} \$ 16$ \\
\hline G17 & СУММ(С7:С9) & $\$ \mathrm{G} \$ 17=\$ \mathrm{I} \$ 17$ \\
\hline G18 & СУММ(D7:D9) & $\$ \mathrm{G} \$ 18=\$ \mathrm{I} \$ 18$ \\
\hline G19 & СУММ(E7:E9) & $\$ G \$ 19=\$ I \$ 19$ \\
\hline $\mathrm{G} 20$ & СУММ(I13:I15) & $\$ G \$ 20=\$ I \$ 20$ \\
\hline G21 & СУММ(I16:I19) & $\$ G \$ 21=\$ I \$ 21$ \\
\hline
\end{tabular}

Стоимость перевозок в условных единицах записываем в блок ячеек В13:Е15 (табл. 3).

Коэффициенты при неизвестных, равные единицам, заносим в ячейки В19:Е21.

В ячейки I13:I15 записываем количество грузов, находящихся в пунктах отправления $\mathrm{A}_{\mathrm{i}}$, а в ячейки
I16:I19 заносим количество грузов, требуемых по заявкам пунктов назначения $\mathrm{B}_{\mathrm{j}}$.

В ячейку G7 записываем условия целевой функции:

СУММПРОИЗВ(В7:Е9;В13:Е15)

Таблица 3

Электронная таблица решения транспортной задачи

\begin{tabular}{|c|c|c|c|c|c|c|c|c|c|}
\hline $\begin{array}{l}\text { № } \\
\text { стр. }\end{array}$ & A & B & $\mathrm{C}$ & $\mathrm{D}$ & $\mathrm{E}$ & $\mathrm{F}$ & G & $\mathrm{H}$ & I \\
\hline 1 & 2 & 3 & 4 & 5 & 6 & 7 & 8 & 9 & 10 \\
\hline 1 & & & & & & & & & \\
\hline 2 & & & \multicolumn{5}{|c|}{ Транспортная задача } & & \\
\hline 3 & & & & & & & & & \\
\hline 4 & & & & & & & & & \\
\hline 5 & & \multicolumn{4}{|c|}{ Значения переменных } & & & & \\
\hline 6 & & $\mathrm{X}(\mathrm{i}, 1)$ & $X(i, 2)$ & $\mathrm{X}(\mathrm{i}, 3)$ & $\mathrm{X}(\mathrm{i}, 4)$ & & Целевая функция Z & $\min$ & \\
\hline 7 & 35 & 10 & $\mathbf{0}$ & 25 & $\mathbf{0}$ & & 620 & y.e. & \\
\hline 8 & 45 & 20 & $\mathbf{0}$ & $\mathbf{0}$ & 25 & & & & \\
\hline 9 & 50 & $\mathbf{0}$ & 10 & 40 & $\mathbf{0}$ & & & & \\
\hline
\end{tabular}


Продолжение табл. 3

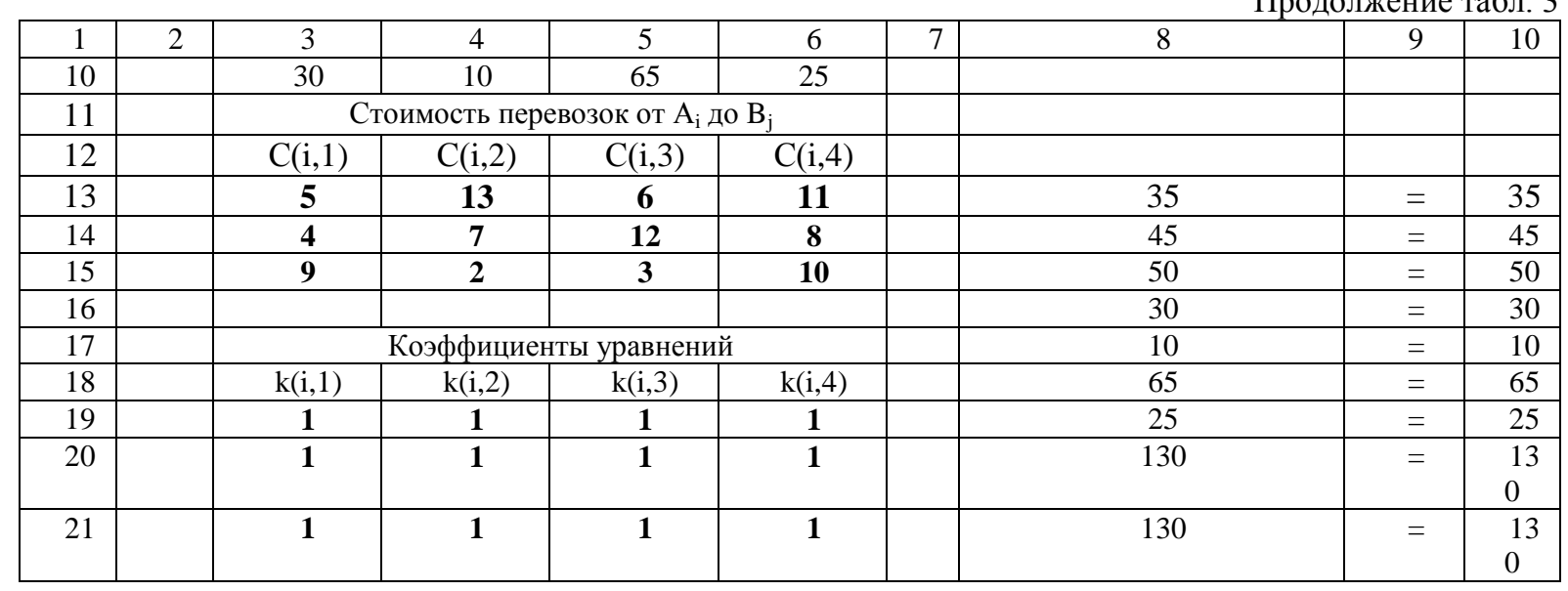

В результате поиска решения найден оптимальный план перевозок, представленный в ячейках В7:E9 табл. 3, то есть сколько, откуда и куда надо перевезти единиц груза с минимальными затратами, при этом целевая функция составила:

$$
Z=620 \text { y.e. }
$$

\section{СПИСОК ЛИТЕРАТУРЫ}

1. Кондратьев Ю. Н. Оптимизация транспортных перевозок в среде EXCEL-7 // Новые технологии и устойчивое управление в лесах северной Европы: Тез. докл. межд. конф. Петрозаводск: Изд-во ПетрГУ. 2001. С. 66. 